\title{
Recent developments in polyurethane-based conducting composites
}

\author{
J. Njuguna ${ }^{\dagger *}$ and K. Pielichowski \\ Department of Chemistry and Technology of Polymers, Cracow University of Technology, \\ Ul. Warszawska 24, 31-155 Krakow, Poland.
}

† On leave from City University, London, School of Engineering, Northampton Square, London, EC1V 0HB, UK.

*Corresponding author. njugunajak@yahoo.co.uk, Tel.+ 4812 6282695, Fax: +48 126282038 (J. Njuguna). 


\begin{abstract}
Polyurethane-based conducting composites with polyaniline, polythiophene or polypyrrole are in the class of modern macromolecular materials that combine the toughness and elasticity of polyurethane matrix with conductivity of intrinsically conducting polymers. Since the methods of preparation strongly influence the structure and properties of resulting composite/blend, this works aim at systematic description of polyurethane based conducting composites. This review has been structured to present an overview on preparations, characterisation and applications of polyurethane-based conducting materials with polyaniline, polythiophene or polypyrrole.
\end{abstract}

Keywords: Polyurethane, conducting polymer, elastomer, polyaniline, polythiophene, polypyrrole 


\section{Introduction}

Polyurethane is a unique material that offers the elasticity of rubber combined with the toughness and durability of metal. Polyurethane (PU), composed of a polyether or polyester soft segment and a diisocyanate-based hard segment, are well-known tough materials and are usually used as an additive to enhance toughness of brittle materials as well as thermal properties [1-5]. The most common diisocyanate are presented on Table I along with that of schematic representation of segmented PU - Scheme 1.

\section{Table I.}

Scheme 1.

Because of the incompatibility between the hard segments and the soft segments, PU undergoes microphase separation resulting in a hard-segment domain, soft-segment matrix, and urethanebonded interphase. The hard-segment domains act as physical cross-links in the soft-segment matrix. The primary driving force for phase separation is the strong intermolecular interaction of the urethane units, which are capable of forming intermolecular hydrogen bonds.

Owing to such interactions, interconnected or isolated hard segments remain distributed in the soft segment matrix, though the soft domain may contain some hard segments dissolved in it, which is evident from the hydrogen bonding of the urethane-NH groups with the oxygen of the ether or ester linkages [6]. These kinds of PU are utilized mainly as water dispersions (coatings, adhesives) and also as biomedical devices, temperature-sensing elements, polymer electrolytes, etc. The recent technological interest concerns the studies on composites containing conductive polymers and an inert polymer matrix. The potential usage of conducting polymer blends includes electro-chromic and electro-optical devices, packaging materials, actuators, batteries, and catalysis. Such elastomers may also have applications as rollers used in electrostatic imaging, cables, EMI-shielding gasket materials, and, potentially, chemical and/or biological sensors [1-6].

In the case of conducting polymers, the objective is the preparation of polymeric materials with good mechanical properties and processability associated with high conductivity or electrochromism [7-10]. Conductive polymers may be synthesised by any or combination of the following techniques: chemical polymerisation, electrochemical polymerisation, photochemical polymerisation, metathesis polymerisation, concentrated emulsion polymerisation, inclusion polymerisation, solid-state polymerisation, plasma polymerisation, pyrolysis or soluble precursor polymer preparation [11-15]. The incorporation of functional groups (e.g. carboxylic, sulfonic, 
phosphonic groups) in the PU backbone, able to react further covalently or non-covalently, leads to PU-ionomers with the improved characteristics such as adhesion, dyeability and ionic conductivity or polymers with special properties like haemocompatibility, polymers with shape memory effects and non-linear optic properties.

Diaz [16], Evans [17], Margolis [18], MacDiarmid and Maxfield [19] and Chandler and Pletcher [20] have comprehensively reviewed developments in conductive polymers. Other reviews include those by Ray and Okomoto [12], Dai and Mau [21], Deronzier and Moutet [22], Heinze [23], Kumar and Sharma [11], Imisides et al. [24] and Inzelt et al. [25], etc. Several excellent reviews have been published in the recent years, specifically dedicated to polyaniline [8-15] and polypyrrole [26] only but none of such solely dedicated to polythiophene has been cited. Vastile applications for conducting polymers are well presented by Kumar and Sharma [11] hereby presented in a modified form in Fig. 1, as well as comparison of their conductivities in Table II.

Fig. 1

Table II

This review has been structured to present an overview on preparations, characterisation, applications and concluding remarks on PU composites/blends with polyaniline, polythiophene or polypyrrole.

\section{Synthesis methodology}

One of the methods of preparation of conducting composites is the mechanical mixing where the controlling factors for a good conductive composite are the sizes and uniformity of particles as well as the efficiency of mixing [1,27]. Another way to prepare the composite is the chemical or electrochemical synthesis in organic solvents [13,14]. Since the organic solvents may cause environmental pollution, a trend to replace the organic solvents by supercritical carbon dioxide or iodine is observed [28]. These methods are briefly discussed here while further insight can be sought elsewhere in the literature [11-15]. Table III presents the synthetic process and characterisation techniques utilised for quick reference.

Table III 


\subsection{Chemical process}

PU-based conducting polymers matrices blends and composites can be synthesized via a number of approaches. These synthetic methods for polymerisation can be mainly classified into chemical and electrochemical synthesis, the chemical approach being the most preferred route mainly due to its simplicity and inexpensiveness in comparison to electrochemical synthesis. Yet, for in situ spectroscopic studies, the electrochemical method is better suited as it holds several advantages in that the synthesis is cleaner, conducting polymer blends and composites are supplied in the form of freestanding films, and also that the electrical properties of the composites can be modified by simply varying the conditions of electrolysis. However, it suffers from the disadvantage that the size of the composite film prepared depends on the size of the electrode. Further, an appropriate matrix polymer-electrolyte system should be available for the penetration of the ionic species (monomer and electrolyte) into the polymer matrix.

The chemical synthesis can be further classified in to chemical in situ polymerisation, counterioninduced processibility, melt processing, dry- and solution-blending, and, emulsion- and dispersion polymerisation, while the electrochemical synthesis involves cyclic voltammetry, potentiostaticand galvanostatic polymerisation [15]. The chemical in situ polymerisations involve an intimate mixing of two components and are one of the useful methods for the in situ polymerisation of new materials.

Likewise, counterion-induced approach produces attractive mechanical properties of the polyblend materials as presented by $\mathrm{Li}$ et al. [15]. The properties of these polymer blend materials may be contrasted with those obtainable using filled polymers, i.e. bulk polymers filled with conducting particles such as carbon black or intractable particles of conducting polymers. For polymer blends made by using the counterion-induced processibility of conducting polymer, the conductivity increases continuously, at remarkably low levels, as a function of the fraction of the conductive polymer complex. This may be compared with the known behaviour of filled polymers, which exhibit a percolation threshold at a volume fraction of about $16 \%$. The smooth onset of conductivity in these polyblend materials indicates an unusual morphology with connected pathways even at remarkably low volume fractions of the conducting complex. Since the mechanical properties remain essentially equivalent to those of the host polymer, the morphology normally consists of an interpenetrating network e.g. a presentation by Banerjee [29] from studies on electrically conductive interpenetrating network composites of polyaniline and carboxymethylcellulose. Counterioninduced processibility of conducting polymer has created a class of stable and processible polymer blends in which the electrical conductivity can routinely be controlled from $10^{-10} \mathrm{~S} / \mathrm{cm}$ to $100 \mathrm{~S} / \mathrm{cm}$ 
in materials, bestowing advantages in processing and with the mechanical properties of the bulk polymers.

Emulsion polymerisation of macromolecular conducting systems is a compartmental polymerisation reaction - taking place in a large number of reaction locally dispersed in a continuous external phase. The reaction is carried out in heterogeneous systems, usually with both aqueous and nonaqueous phases. The monomer as well as the polymer usually belongs to the non-aqueous phase. In inverse systems, water-soluble monomers are dispersed in a non-aqueous medium. A typical emulsion polymerisation consists of $30 \%$ monomer, $65 \%$ water, with the rest being emulsifier (surfactant), initiator and other additives. Emulsification initially results in micelles swollen with solubilized monomer and surfactant-stabilized monomer droplets. As the initiator decomposes, a new phase appears consisting of the latex particles, which contain macromolecules of a fairly high degree of polymerisation swollen with monomer and stabilized by the surfactant.

The advantage of compartmentalized polymerisation technique over other polymerisation processes is that the molecular weight of the polymer can easily be controlled by the addition of chain transfer agents; thus there can be control over the properties of the final product. Dispersion polymerisation may be defined as the polymerisation of a monomer dissolved in an organic liquid or water to produce an insoluble polymer in the form of a stable colloidal dispersion. The colloidal stability of the resulting particle is provided by the adsorption of an amphilic polymeric stabilizer or a dispersant, which is present in the organic medium on the surface of the polymeric particles. Hence, this process may also be viewed as a kind of precipitation polymerisation in which flocculation is prevented and the particle size is controlled.

Conducting and insulating polymers can also be dissolved in a common solvent and afterwards processed into thin films, a process known as solution blending. In contrast, blending the powders of polyaniline, polypyrrole, polythiophene etc. and the host polymer in a mixer may prepare dry blends. While in melting process, the conducting polymer is dispersed in a thermoplastic polymer matrix by mechanical mixing and then compression moulded in a hot press. A major requirement for this process is that the thermal stability of the conducting polymer should be sufficient to withstand compounding in the melt of the chosen polymer host. For this purpose, the host polymer should possess a low melting temperature.

\subsection{Electrochemical process}

Electrochemical synthesis is cleaner and the polymer composite film can easily be peeled off from the electrode and washed. Simply changing the electrolytic conditions can vary the electrical 
properties of conducting polymer - insulating polymer films. By electro-oxidative polymerising the conducting polymer on a non-conducting, mechanically superior polymer film-coated electrode, a composite film having a high conductivity and the good mechanical properties of the polymer substrate may be obtained. The advantage of the electrochemical route is that the conducting polymer composite is obtained in the form of a freestanding film, which is often needed for technological applications. In the electrochemical synthesis, the monomer, the solvent and the electrolyte anion diffuse into the insulating polymer coating. As a result, polymerisation starts in the interface between the electrode surface and the polymer film.

The electrochemical synthesis is generally accomplished by potentiostatic or galvanostatic polarization. Alternatively, the synthesis may also be carried out by cyclic voltammetry, driving the electrode potential between the limits of the monomer oxidation and reduction of the synthesized electroactive-conducting polymer. For instance, in Csaho'k et al. [30] investigations, polyaniline (PANI) was deposited on two adjacent or on all bands by electropolymerisation from $0.2 \mathrm{~mol} / \mathrm{dm}^{3}$ aniline solution in $1 \mathrm{~mol} / \mathrm{dm}^{3} \mathrm{HClO}_{4}$ or $\mathrm{H}_{2} \mathrm{SO}_{4}$ solutions, respectively, under continuous potential cycling between -0.2 and $+0.75 \mathrm{~V}$ (saturated calomel electrode) at a scan rate of $100 \mathrm{mV} / \mathrm{s}$. Here a platinum wire was used as a counter electrode, while the reference electrode was a saturated calomel electrode (SCE). After polymerisation, the polymer-coated electrode was rinsed with the supporting electrolyte solution that was used for the further experiments. Before the in situ conductivity measurements, the films were oxidized and reduced 20-30 times by cycling in order to remove traces of aniline and oligomers. The total amount of the polymer on the surface was determined from the area under the cyclic voltammetric waves in a slow sweep rate experiment.

\section{Synthesis of TPU-PANI composites}

Polyaniline is the only conducting polymer whose electrical properties can be controlled suitably by charge-transfer doping and/or protonation. Due to its reversible electrochemical response during anodic oxidation and cathodic reduction, it is useful as a secondary electrode in rechargeable batteries and electrochromic display devices. However, two major limitations of conducting polyaniline are an inability to process it by conventional methods and its poor mechanical properties. These limitations can be overcome by preparing conducting PANI blends and composites, which possess the mechanical properties of the insulating host matrix and the electrical properties of the conducting PANI guest [7,31]. 
Recently, PANI was found to exhibit unusual chemical, electrical, and optical phenomena, both in insulating and conducting forms. Incorporation of conductive PANI into a hosting polymer substrate forming interpenetrated networks (IPN) has been a newly adopted method as an approach to combine electrical conductivity of PANI with desirable mechanical strength of insulating polymers. These unique properties make PANI useful in many applications, particularly in energy storage, electronics, photovoltaic devices, displays, and sensors, antenna material in cellular phone, as an antistatic paper roller in a printer, and as a fragile, preventive wrapping bag for packing electronic products [32].

The unique properties have led to an interest in the potential use of PANI as a new class of conductors. This interest was generated due to the relative ease of synthesis, low cost, and the stability of PANI in air. In spite of all, the insulating form of PANI, polyaniline emeraldine base (PANI-EB), suffers from limited solubility in organic solvents. This difficulty was recently resolved by Cao et al. [33] by functionalising the dopant counter-anion with polar and non-polar analogs to increase solubility in organic solvents. The aniline polymers are also on beam light because of their environmental stability, controllable electrical conductivity and interesting redox properties associated with the chain nitrogen. These polymers also exhibit crystallinity and solution- or counterion-induced processability.

Pei and $\mathrm{Bi}$ [34] used potentiostatic polymerisation technique to prepare polyaniline-polyurethane composite films with high flexibilities and mechanical strengths similar to those of pure polyurethane and conductivities close to that of pure PANI. This was accomplished by electropolymerisation aniline on a PU-coated platinum electrode in a water/acetonitrile/ethylene glycol electrolyte solution at a constant potential of $2.0 \mathrm{~V}$. In the composite films, polyaniline was dispersed in a particulate form inside and on the inner surfaces of the polyurethane substrates. It was found that the electropolymerisation reaction of aniline proceeds much faster in basic solutions. The composite films thus prepared showed higher conductivities than those prepared in acid solutions.

Later on, Sawai et al. [35] used the same synthetic process previously employed by Pei and Bi [34] to prepare PANI-PU conducting composites. However, the polyaniline-polystyrene sulphonate composite films used in this study were prepared in an aqueous solution containing $1 \mathrm{M} \mathrm{HCl}$ and $0.5 \mathrm{M}$ aniline at a constant potential of $0.75 \mathrm{~V}$ vs. $\mathrm{Ag} / \mathrm{AgCl}$. Elsewhere, Wang et al. [36] prepared PANI-PU IPN using chemical oxidative process in $\mathrm{HCl}$. The resulting products were then washed with water followed by tetrahydrofuran (THF) to remove an excess of aniline monomers, PANI with a low molecular weight and persulfate salts. The residual polyaniline at its emeraldine base form, physically attached to the surface of the product was cleaned by repeatedly rinsing with $\mathrm{N}$ methyl-2-pyrrolidinone (NMP) and THF. 
In another development, Yang and Lee [37] have reported on polyaniline polyacid complexes blended with water-based PU of different chemical structures. On their experiment, 0.03 mole of freshly distributed aniline was added in to aqueous solution of polystyrene sulfonic acid (PSSA), polyacrylic acid (PAA), or poly (2-acrylamido-2 methyl-1-propanesulfonic acid) (PMPSA) containing 0.03 mole monomer units. 0.0075 mole of ammonium peroxydisulfate was then added with vigorous stirring. The solution was observed to turn green after a few minutes. Several hours later, the solution was dialyzed in an acidic medium to remove unreacted aniline monomer and soon after the product was dried. Finally, the polymer blends were made by mixing the aqueous solution of polyaniline polyacid complexes ( $\mathrm{pH}$ of the solution being adjusted to 8) and water based PU in various ratios. The mixture was then coated on the glass plate and then evacuated under vacuum at $50^{\circ} \mathrm{C}$ for 24 hours.

Flexible and freestanding films from polyurethane based on castor oil were synthesized by Malmonge et al. [38]. The PU based on castor oil was prepared by pre-polymer method while the stock solutions of PANI dissolved in NMP were separately prepared. These polyaniline solutions were mixed at the desired proportions with the polyol and then with the pre-polymer previously dissolved in NMP. Films (30-100 $\mu$ m thick) were prepared by casting these solutions on to glass surface by immersion in hot water.

Špírková et al. [39] first prepared polyaniline (emeraldine) sulfate by oxidative polymerisation of aniline sulfate $(0.1 \mathrm{M})$ at $0-2^{0} \mathrm{C}$ using ammonium peroxodisulfate $(0.2 \mathrm{M})$ as oxidant. The precipitate was washed repeatedly with $0.1 \mathrm{M}$ sulfuric acid, followed by acetone and later dried in vacuum at $60^{\circ} \mathrm{C}$. The PU network was then prepared from trihydroxypoly- (oxypropylene) (PPT) and diisocyanate diphenylmethane (MDI, mixture of 2,4'- and 4,4'-isomers). The dibutyltin dilaurate (DBTDL) was used as the catalyst for the urethane formation. The PANI powder, PPT and DBTDL were first treated in ultrasonic bath for reduction of PANI particle aggregation, and then stirred at $50^{\circ} \mathrm{C}$ at a reduced pressure for residual moisture and air removal. The MDI was then added at room temperature into a mixture containing 1, 5, and $10 \mathrm{wt} . \%$ of PANI. DC voltage was then applied to the polymerisation mixture placed between aluminium blocks separated by silicone rubber spacer and kept at $90{ }^{\circ} \mathrm{C}$ for 24 hours.

Liao et al. [40] reported a series of novel conducting IPN prepared by sequential polymerisation of maleimide-terminated polyurethane (UBMI) and PANI that was doped with dodecylbenzenesulfonic acid. The solution of PANI doped with dodecylbenzenesulfonic acid (PANIDB) and maleimide-terminated PU at various weight ratios in chloroform was placed in a flask and then stirred for 48 hours with the aim of approaching equilibrium conditions. Then a solution of benzoyl peroxide, 1 wt.\% based on the weight of the maleimide-terminated PU, was added to the flask and stirred well at room temperature. The mixture was later casted into a mould 
at the same temperature. After the solvent was allowed to evaporate for 10 hours, the resultant mixture, formed as a film, was cured at $70^{\circ} \mathrm{C}$ for 10 hours and then post-cured at $120^{\circ} \mathrm{C}$ for three hours. The samples were then conditioned in desiccators at 50\% relative humidity for at least 3 days before subjected to testing. This work followed a previous one in which a novel urethanemodified bismaleimides (UBMI) was synthesized via the imidization of the natural clay organically terminated PU prepolymer and maleic anhydride [41].

Up to $12.5 \%$ PANI have been conveniently filled to PU/ poly (methyl methacrylate) PMMA (50/50) IPN system by Siddaramaiah [42]. The PU prepolymer was reacted with methyl methacrylate (MMA) containing $0.5 \%$ benzoyl peroxide. The content of the flask were mixed thoroughly for $10 \mathrm{~min}$ and later was stirred constantly with the addition of different percentage of emeraldine base (EB), which had been already mechanically mixed with camphour sulphonic acid (CSA) using an agate mortar and pestle. Finally, 1\% dibutyl tin dilaurate (DBTL) and 1\% ethylene glycol dimethacrylate (EGDM) were added as a catalyst and as a crosslinker, respectively. The homogeneous mixture was poured into a glass mould sprayed with the releasing agent. The mould was kept at room temperature for $24 \mathrm{~h}$ for polymerisation of PU; the temperature was then slowly raised to $80^{\circ} \mathrm{C}$ to initiate MMA polymerisation for $12 \mathrm{~h}$ followed by further maintenance at $110^{\circ} \mathrm{C}$ for $10 \mathrm{~h}$.

It has been reported that PU with m-phenylene 4-diaminosulfonic acid (PDSA) as chain extender can deeply influence the properties of its blends with $n$-dodecyl benzene sulfonic acid (DBSA) doped polyaniline (PADB). The sulfonic chain extender PDSA provides an additional probability of creating H-bonding with PADB molecules that can be characterized by IR spectra. In one of such investigation [32], PU prepolymer was prepared with 2:1 mole ratio of purified MDI to polyol polytetramethylene oxide (PTMO) at $70^{\circ} \mathrm{C}$ under nitrogen gas. The obtained PU prepolymer was mixed with equal amount of moles of chain extender (1.4 butanediol (BD) or PDSA) at $70^{\circ} \mathrm{C}$ until the isocyanate (-NCO groups) disappear in the IR spectrum, followed by drying in vacuum oven for 3 days. A PU solution in mixed solvents of chloroform and N, N dimethyl formamide (DMF) at a ratio of 1:2 was blended with DBSA-doped polyaniline chloroform solution at various compositions. The obtained polymer blend solution was stirred at least $24 \mathrm{~h}$, followed by casting in a petri dish and dried in hood followed by further drying in a vacuum oven for at least $24 \mathrm{~h}$.

While Pei and Bi [43] also studied electro-oxidative polymerisation of aniline on platinum anodes coated with PU film, Wang et al. [6] investigated on conducting PU, aniline-terminated polyaniline oligomeric powders (OPA) dissolved in NMP. The resulting PU prepolymer in NMP was mixed with an equivalent amount of OPA-NMP mixture plus a chain extender $\mathrm{BD}$ at $70^{\circ} \mathrm{C}$ until the isocyanate disappeared, followed by drying in a vacuum oven for 3 days. The molar ratio of the OPA relative to the chain extender was varied to control the content of the aniline containing 
urethane-urea blocks in the copolymers but keeping the total amount equal to that of PU prepolymers. The PU prepolymer presented on Scheme 2 was prepared with a 2:1 mole ratio of purified MDI to polyol (poly(butylene adipate) glycol) (PBA) with a molecular weight of 1000 at $70^{0} \mathrm{C}$ under nitrogen gas.

Scheme 2.

\section{Preparation of TPU-PPy composites}

Polypyrrole (PPy) has been extensively studied because of its very high conductivity in the doped state and easy chemical or electrochemical polymerisation, which make it a good candidate for many applications, e.g. sensor, biosensor, modified electrodes, actuators, and electronic devices $[44,45]$. Conversely, PPy has poor mechanical properties and poor stability at ambient conditions, which restricts its utilization for different commercial applications. As well, PPy like many other conductive polymers is insoluble in most organic solvents and infusible because of its decomposition before melting. As a result, the conventional methods for polymer processing, such as melt processing and solution casting, may not usually be applied to this material.

Due to these reasons, considerable research has been undertaken in improving its stability and mechanical properties as well as its processability. Several attempts have been made to improve the poor mechanical properties of conductive polymers by making blends or composites with other conventional polymers e.g. PPy-poly(methyl methacrylate) [46,47], PPy-poly(vinyl alcohol) [48], PPy-poly(vinyl chloride) [49], PPy-poly(ethylene terepthalate) [50], and PPypoly(tetrafluoroethylene) [51]. One of the beaming applications of TPU-PPy composites is on electrodes for bending-electrostrictive polyurethane actuator reported by Watanabe et al. [52]. The wrinkled electrode was prepared through in situ deposition of polypyrrole onto the polyurethane elastomer film that was being uniaxially drawn. After the deposition, the film was released from the drawing to make the electrode wrinkle.

Reece and co-workers [53] adopted the electrochemical preparation of PPy colloids using the same PU/poly(ethylene oxide) latexes as previously employed by Wiersma et al [54]. Polymerisations were performed using solutions containing either nitrate or 4,5-dihydroxy-1,3-benzenedisulfonic acid (DBDA) as dopants. The latter dopant was considerably of interest due to its known electrocatalytic properties with respect to the electrosynthesis of PPy as previously reported by Zinger [55,56]. An aqueous anolyte solution $(100 \mathrm{ml})$ containing $0.15 \mathrm{M}$ pyrrole $(1 \% \mathrm{w} / \mathrm{v}), 0.1 \mathrm{M}$ DBDA and 4\% w/v PU, and an aqueous catholyte solution $(250 \mathrm{ml})$ containing $0.1 \mathrm{M} \mathrm{NaNO}_{3}$ were employed. The anolyte solution was pumped through the reticulated vitreous carbon (RVC) anode 
at $40 \mathrm{ml} / \mathrm{min}$, while the catholyte solution was pumped through both RVC cathodes also at a flow rate of $40 \mathrm{ml} / \mathrm{min}$. A constant potential of $0.45 \mathrm{~V}$ was applied for the first $2 \mathrm{~h}$ of the reaction, followed by $0.60 \mathrm{~V}$ for a further $2.5 \mathrm{~h}$. Samples were withdrawn at regular intervals within the experiment for UV-Vis and HPLC analysis. At the completion of the synthesis the solution was left to stand for $24 \mathrm{~h}$, which was then dialysed against water using cellulose dialysis tubing for a further 24 hours.

Wiersma et al. [54] noted the chemical polymerisation of pyrrole or aniline in the presence of a nonionic stabilised resin dispersion results in stable hybrid dispersion. The authors synthesised polypyrrole-PU starting with the preparation of a water solution of $\mathrm{Fe}\left(\mathrm{NO}_{3}\right)_{3} \times 9 \mathrm{H}_{2} \mathrm{O}$. A solution of pyrrole (vacuum distilled) in distilled water was also prepared. Both solutions were added to nonionic stabilised dispersion of polyurethane in water. After 20 hours stirring at $20^{\circ} \mathrm{C}$, the dispersion was centrifuged for one hour at $14000 \mathrm{rpm}$ and the sediment redispersed in demineralised water. The particles were statically stabilised by poly(ethylene oxide) grafted onto the original PU latex taking advantage of colloids combination with the film-forming ability of PU with the conductivity of PPy. The authors also suggested that this particular procedure could also be applied to PANI-PU composite synthesis.

The effect of conducting PPy on the morphology and ionic conductivity of thermoplastic polyurethane (TPU) doped with $\mathrm{LiClO}_{4}$ has been described by Wen et al. [57] and compared with those of pure PU system. The PU-PPy composites were prepared by chemical polymerisation of pyrrole inside PU films. Bi and Pei [58] has reported such composites of polyurethane and polypyrrole whereby ester-based polyurethane for making the composites were prepared by electrochemical method.

Unlike Bi and Pei [58], Wen et al. [57] used polyether-based PU that has ether (-O-) groups in the main chain; the polyurethane has the added advantage to coordinate alkali metal ions and can be used as polymer electrolyte in Li batteries. PU films $(100-200 \mu \mathrm{m})$ were obtained by coating from a $10 \%$ solution in DMF on polypropylene (PP) plates. Most of the solvent was evaporated under vacuum at $50^{\circ} \mathrm{C}$ and then dried under vacuum at $100^{\circ} \mathrm{C}$ for $72 \mathrm{~h} . \mathrm{FeCl}_{3} \times 6 \mathrm{H}_{2} \mathrm{O}$ was dissolved in propylene carbonate (PC) to make $1.75 \mathrm{M} \mathrm{FeCl}_{3} / \mathrm{PC}$ solution. The TPU films were then immersed into $1.75 \mathrm{M} \mathrm{FeCl}_{3} / \mathrm{PC}$ solutions for one hour. After that the $\mathrm{FeCl}_{3}$ impregnated films were taken out from the solutions, excess solution was soaked with filter paper and then dipped into $0.75 \mathrm{M}$ pyrrole/PC solutions at $25^{\circ} \mathrm{C}$ for $6 \mathrm{~h}$. The composite films were then withdrawn and washed with water by soaking and replacing several times with fresh water for complete removal of unreacted $\mathrm{FeCl}_{3}$. After removing the unreacted $\mathrm{FeCl}_{3}$, the composite films thus obtained were dried under vacuum at $70^{\circ} \mathrm{C}$ for 1 week. 
A process that could be used for conductive dense elastomers synthesis has been proposed by a few reports [59-62] though these researchers main work was aimed at producing conductive PU foams. In fact few experiments have also been carried out to assess the feasibility of producing conductive polymers from dense elastomeric films of PU/polybutadiene (PBD) and PU/poly(styrene-co-ranbutadiene) (SBR) [59]. To start with, He et al. [61,62] investigated the properties of PU-PPy foam by vapour-phase polymerisation and correlated the effects of temperature, types and concentration of oxidants and time of polymerisation on the conductive properties of the composite foam.

$\mathrm{Fu}$ et al. [60] reported the synthesis of conductive PU foams by diffusing a methanol solution of $\mathrm{FeCl}_{3}$ into the foam and then exposing the PU/oxidant composite to pyrrole vapour. A deficiency of that process, however, was that rather large quantities of methanol, a volatile organic chemical (VOC), were used to introduce the oxidant into the PU foam and to remove unreacted oxidant and redox by-products. In later work, Shenoy and co-researchers [59] showed that the volatile products could be eliminated by replacing the methanol with supercritical carbon dioxide $\left(\mathrm{scCO}_{2}\right)$ or a mixture of $\mathrm{scCO}_{2}$ and ethanol. Polymer synthesis and processing using supercritical $\mathrm{CO}_{2}$ is a growing research specially because the supercritical $\mathrm{CO}_{2}$ solvent has a low critical temperature $\left(\mathrm{T}_{\mathrm{c}}\right.$ $\left.\sim 31^{\circ} \mathrm{C}\right)$ and moderate critical pressure $\left(\mathrm{P}_{\mathrm{c}} \sim 7.4 \mathrm{MPa}\right)$, is relatively inexpensive and environmentally friendly [63].

With such considerations, the follow-up work [59] reported a conductive PU/PPy composite elastomer that was prepared using $\mathrm{scCO}_{2}$ to impregnate $\mathrm{PU}$ foam with ferric triflate or ferric trifluoroacetate, followed by an in situ polymerisation of pyrrole. The PU foam was used as supplied while supercritical fluid extraction (SFE) was used for impregnating the foams with oxidant. In a typical experiment, $250 \mathrm{mg}$ of oxidant and magnetic stir bar were placed in the SFE vessel with the oxidant. The ethanol concentration was varied between 0.2 and 1.3 vol.\% and the experiment was run at $35^{\circ} \mathrm{C}$ and a pressure of $17.2 \mathrm{MPa}$.

Though this approach was adequate, it necessitated the synthesis of $\mathrm{CO}_{2}$-phillic, fluorinated metal carboxylate and sulfonate oxidants, and solubility was generally low. Shenoy and co-workers have recently proposed a solvent-free method for preparing electrically conductive polyurethane foams and elastomeric films [28]. The method consists of impregnation of the oxidant into the foam through the sublimation of iodine, followed by in situ polymerisation of pyrrole using pyrrole vapours to obtain a polypyrrole/polyurethane foam composite. The authors employed $\mathrm{I}_{2}$ for the in situ polymerisation of pyrrole within PU foam. $\mathrm{I}_{2}$ readily sublimes, thus allowing for the impregnation of foam with a vapour, and the vapour condenses within the foam, which is then exposed to pyrrole vapours to initiate polymerisation resulting PU-PPy composite foams. 


\section{Preparation of TPU-polythiophene composites}

Recently, Sari and co-researchers synthesised polyurethane/polythiophene (PU-PT) conducting copolymers using PU as an insulating matrix by electrochemical method (suspension method and dropping-coating method) [64]. In dropping coating method, PT/lithium perchlorate $\left(\mathrm{LiClO}_{4}\right)$ and $\mathrm{PT} /$ tetraethylammonium tetrafluoroborate $\left(\mathrm{Et}_{4} \mathrm{NBF}_{4}\right)$ were chosen in order to synthesize PU-PT bipolymer films in acetonitrile and benzonitrile medium, respectively. The PU film was coated on the cleaned electrode surface from a 1-g/100 ml chloroform solution of PU. The coating was carried out by dropping the of above solution onto each surface of the working electrode. The PU-coated electrode was placed into the electrolysis cell and the electrolysis was run in a given potential. The amounts of insulating and conducting polymers were determined gravimetrically.

For the suspension method [64], the PU/PT bipolymer films were synthesized onto a platinum electrode surface using a mixture of $0.1 \mathrm{M}$ thiophene $+0.2 \mathrm{M} \mathrm{LiClO}_{4}+\mathrm{PU}$ solution in each halfcell in an acetonitrile medium. Prior to electrosynthesis, the mixture was thoroughly stirred to disperse PU solution. PU/PT bipolymer film in benzonitrile medium was also achieved using a mixture of $0.1 \mathrm{M}$ thiophene $+0.2 \mathrm{M} \mathrm{Et}_{4} \mathrm{NBF}_{4}+\mathrm{PU}$ solution in each half-cell under the same conditions.

Urethane-substituted polythiophene exhibits good solubility and improved processability. A good example is the PU-polythiophene elastomer reported to display enhanced optical properties such as nonlinear optical effects, thermochromic and solvatochromic behaviour as well as outstanding EMI shielding properties $[65,66]$. In this work, the urethane-substituted polythiophene in the $\beta$-position (P3UT) that was chemically synthesized by solution polymerisation using $\mathrm{FeCl}_{3}$ as the oxidant in chloroform, is not only soluble in common organic solvents, such as chloroform and tetrahydrofuran, but also in strong polar solvents such as $\mathrm{N}, \mathrm{N}$-dimethylformamide, N, Ndimethylacetamide and NMP. The urethane-substituted monomer was synthesised by reacting 3(2thienyl)ethanol with butyl isocyanate in toluene. The blends of P3UT with PU were prepared by dissolving each component in to THF solvent. The film were casted from these solutions in to a glass round dish but the final films were also washed with acetone before use $[65,66]$.

Ruckenstein and Sun [67] prepared PU films containing 3-methylthiophene by condensation of poly(propylene glycol) diol, poly(propylene glycol) triol and toluene 2,4-diisocyanate in the presence of a catalyst and 3-methylthiophene (3-MT) at room temperature through overnight. The immersion of the films in a suitable organic solution of ferric chloride led to their rapid coating with conductive layers of poly(3-methylthiophene) via the diffusion-oxidative reaction of 3methylthiophene and ferric chloride. In a typical experiment, poly(propylene glycol) triol, poly(propylene glycol) diol, and toluene 2,4-diisocyanate, 3-methylthiophene, and butyltin dilaurate 
(catalyst) were well mixed in a tube and then poured on a glass plate where they formed a 3-MT-PU film at room temperature. The film was then peeled away from the glass and immersed into an organic solution of $\mathrm{FeCl}_{3}$ so as to coat the film with a conductive film containing PMT via the oxidative polymerisation in 3-MT.

A relatively new monomer, poly(3,4-ethylenedioxythiophene) (PEDOT), is of considerable interest as it displays superior environmental stability compared to PPy at both ambient and elevated temperatures. This can be partly attributed to the 3,4-disubstituted thiophene rings, which unlike other unsubstituted polyheterocycles leave no sites free for carbonyl formation. These features have resulted in conductivities as high as $210 \mathrm{~S} / \mathrm{cm}$ for electrochemically synthesized PEDOT. It is also noteworthy that, unlike PANI, PEDOT is not susceptible to dedoping by base. In addition, because of its low coloration, PEDOT has also attracted attention as a component in electrochromic devices [68-71].

\section{Properties}

\subsection{Electrical properties}

PANI doped with methane sulphonic acid (MSA) has recently been found by Sanjai et al. [72] to exhibit three-dimensional variable range hopping (VRH) conduction, which is not the case with HCl-doped PANI. The electric field dependence of its conductivity is also reportedly consistent with VRH behaviour. It follows that the conduction process in polyaniline and polypyrrole has been explained by the hopping of electrons along the polymeric chain [73-75]. The charge transport in polyaniline protonated fully with MSA and PANI (MSA)-PU blend were investigated through measurements of temperature and electric field dependence of conductivity, temperature dependence of thermoelectric power and magnetic susceptibility and electron spin resonance at room temperature [72]. The blend follows a one-dimensional VRH type of conduction and the electric field dependence of its conductivity exhibits the Poole-Frenkel effect.

The temperature-dependent magnetic susceptibility measurements indicated the presence of Pauli and Curie spins in both polyanilines protonated fully with MSA and PANI (MSA)-PU blend studied by Sanjai et al. [72]. The percentage of Lorentzian and Gaussian spins was estimated from electron spin resonance measurements. In case of PANI-MSA, a larger number of spins was noted to be delocalised. In another development, Dutta et al. [76] described the d.c conductivity by VRH model whereby the large value of VRH exponent observed $(>0.5)$ indicated that hopping transport 
occurs between the superlocalised states of polymer with the frequency $(\omega)$ dependence of conductivity satisfying the $\omega^{\mathrm{s}}$ power law. The variation of s with temperature suggested that the a.c conduction is due to the correlated barrier hopping $(\mathrm{CBH})$. The onset frequency $\omega^{\mathrm{s}}$ is proportional to the conductivity, $\sigma^{\mathrm{s}}$, while the permittivity increased sharply at low frequency and high temperature.

Liao et al. [40] measured conductivity using the collinear four-point probe method, a method also selected by Ref. $[65,67]$. The polymer film was fastened to wire contacts using the conductivity silver paint. A constant current was applied to the outer electrodes and the resulting voltage across the inner elecrodes was recorded. In a more recent work, the authors measured resistance $(R)$ of the material by a four-probe measurement instrument but this time the conductivity was obtained from the formula, $\mathrm{s}=\mathrm{L} / \mathrm{RA}$, where $\mathrm{L}$ is thickness and $\mathrm{A}$ is cross-section area [6]. The conductivity of the urethane-aniline block copolymers doped with $\mathrm{HCl}$ was recorded lower as compared with that of the neat OPA doped with the same $\mathrm{HCl}$, since the conductive OPA blocks in copolymer is diluted and prepared in the matrix and more restricted in electric transfer than that in the neat OPA. The conductivity of the coating layer in Ref. [67] was calculated from the electrical resistance and the thickness of the coating layer.

Shenoy et al. [59] studies on conductive PU/PPy composite elastomeric foams found that the addition of small amount of ethanol to the $\mathrm{scCO}_{2}$ greatly improves the solubility of the oxidants in $\mathrm{scCO}_{2}$. The conductivity was observed to range from $10^{-7}$ to $10^{-2} \mathrm{~S} / \mathrm{cm}$ depending on how much ethanol in cosolvent was used for incorporating the oxidant. As the concentration of ethanol in the $\mathrm{scCO}_{2}$ was increased from 0 to 13 vol.\%, the amount of oxidant impregnated into the PU foam increased which resulted in a corresponding increase of the amount of PPy produced. In a much more recent work [28], the conductivity was reported to increase rapidly from $\sim 10^{-6}$ to $\sim 10^{-1} \mathrm{~S} / \mathrm{cm}$ as the PPy- $\mathrm{I}_{2}$ concentration increased from ca. 2 to about 40 wt.\%, which is consistent with a percolation process.

Carone et al. [77,78] observed that when elastomeric conducting copolymers prepared by grafting polyaniline (EB) or sulfonated polyaniline (SPAN) chains to the backbone of a carboxylated segmented polyurethane are $\mathrm{HCl}$ doped, their electrical conductivity increases many orders of magnitude, reaching values of about $10^{-3} \mathrm{~S} / \mathrm{cm}$. Stretching the copolymer films may further increase the conductivity, measured along the deformation direction. Electrical current passing through the sample at $\mathrm{U}=33.4 \mathrm{~V}$ was monitored after deposition of gold electrodes on the sides of either parallel to the primary electric field or in the perpendicular direction [39]. In the direction parallel to the primary electric field, conductivity was reported to increase with temperature and with increasing PANI content as expected. As well, the conductivity in the direction of the original field 
was reportedly larger than that in the perpendicular field though the absolute values of conductivities were relatively low $\left(\sigma<10^{-7} \mathrm{~s} / \mathrm{cm}\right)$.

A series of PU elastomers based on toluene diisocyanate, polypropylene glycol $(\mathrm{M}=2050)$ and ethylene glycol were prepared and doped with PANI, $\mathrm{Fe}_{2} \mathrm{O}_{3}$ and $\mathrm{Pb}_{2} \mathrm{O}_{3}$ by Soliman et al. [79]. Electrical resistivity studies on these composites found that the conduction was mainly ionic in nature between 50 and $120^{\circ} \mathrm{C}$. The resistivity decreased with an increase of $\mathrm{Fe}_{2} \mathrm{O}_{3}$ and $\mathrm{Pb}_{2} \mathrm{O}_{3}$ content, indicating the role of transition metal ions. Insertion of PANI increased the d.c. conductivity by three orders of magnitude. For optical studies, reflection spectra were obtained in the range of 400-2500 $\mathrm{nm}$ and analysed in terms of $\mathrm{d}-\mathrm{d}$ transitions. It was concluded that the samples doped with $\mathrm{Fe}_{2} \mathrm{O}_{3}$ and $\mathrm{Pb}_{2} \mathrm{O}_{3}$ have three direct energy band transitions while those doped with polyaniline have two energy band transitions. Also the PANI, $\mathrm{Fe}_{2} \mathrm{O}_{3}$ and $\mathrm{Pb}_{2} \mathrm{O}_{3}$ enhanced the optical properties of all the investigated samples. Meanwhile, the conductivity of the blends made from PU of molecular weight $\sim 100000$ consisting of aliphatic groups containing 5 wt.\% of PANI complexes has been reported to be $\sim 0.5 \mathrm{~S} / \mathrm{cm}$ [37]. The same researchers [37] simultaneously reported the conductivity of PU consisting of some aromatic groups but with the same PANI concentrations to be $\sim 5 \mathrm{~S} / \mathrm{cm}$.

Diaconu et al. [80] investigated the properties of series of polyurethane/polypyrrole composite films by d.c. conductivity, dielectric spectroscopy and tensile strength techniques. A dependence of the physical properties of the composite films on the polyurethane structure, the nature of the oxidizing agent and the polypyrrole content was observed comparatively with the untreated polyurethane cationomer films. Insertion of polypyrrole increases the d.c. conductivity with about six orders of magnitude. Dielectric relaxation spectra are slightly affected by the oxidizing agent but more influenced by the polypyrrole content.

Electrical conductivity in blends of PU/PANI with weight compositions of 90/10 and 80/20 has been reported to reach ca. $10^{-9} \mathrm{~S} / \mathrm{cm}$ and $10^{-4} \mathrm{~S} / \mathrm{cm}$ respectively for doping aqueous solution medium using either $1 \mathrm{M} \mathrm{HCl}$ or p-toluene sulphonic acid (TSA) as the dopant [38]. In the same experiment, conductivity in the order of $10^{-2} \mathrm{~S} / \mathrm{cm}$ was obtained after doping in DMF solution for both blends and dopants. The improvement in doping efficiency was attributed by the authors to swelling effect of the polymeric microstructures by DMF, which makes it easier for the dopant ions diffusion.

In one other development, the conductivity of the gelled electrolytes was measured via impedance analysis with electrochemical cells consisting of the electrolyte film sandwiched between two blocks of stainless steel, sealed with O-ring in a jacketed glass tube where heating/cooling water circulation was made through the outer jacket. The impedance analysis was performed under an oscillation potential of $10 \mathrm{mV}$ from 100 to $1 \mathrm{kHz}$. The conductivity was calculated by $\sigma=l / \mathrm{AR}_{\mathrm{b}}$ 
where $\mathrm{R}_{\mathrm{b}}$ is the bulk resistance from a.c impedance, $l$ is the film thickness and $\mathrm{A}$ is surface area of the electrodes [57].

Some of the conductivity of PU conducting composites/blends presented in this work are analysed in Table IV for quick reference.

Table IV

\subsection{Mechanical properties}

Ruckenstein and Sun [67] did tensile testing on PU films containing 3-methylthiophene at room temperature with a cross-head speed of $20 \mathrm{~mm} / \mathrm{min}$. To overcome the problems relevant to the low processability and scarce mechanical properties of polyaniline, Carone et al. [77] proposed the synthesis of elastomeric conducting copolymers prepared by grafting polyaniline (EB) or sulfonated polyaniline (SPANI) chains to the backbone of a carboxylated segmented polyurethane. In a more recent work [78] the researchers investigated the physical and chemical-physical properties of the copolymers. As evidenced by DSC and DMTA characterization, the introduction of EB or SPANI in the modified PU matrix enhances the hard-soft phase segregation effect, because of the strong tendency of the conductive polymer chains to aggregate. Moreover, the EB and SPANI chains, grafted to the polyurethane backbone, acting as reinforcing filler, give rise, compared with the mechanical properties of the insulating matrix, to an increase of the Young modulus and a decrease of the tensile strength.

Concerning the mechanical properties, the PU/PMMA IPNs were found to possess an average tensile strength of $1.15 \mathrm{MPa}$ [42]. Addition of 2.5\% (PANI)CSA to PU/PMMA IPN did not affect its original tensile strength. Tensile property increased with increase in PANI content above $2.5 \%$ addition. The researchers attributed these effects to reinforcing effect of PANI and the formation of hydrogen bond between -NH of PANI with -NHCOO of PU/PMMA polymer networks [32] as illustrated on Scheme 3.

\section{Scheme 3.}

Wang et al. [6] prepared samples of the urethane-aniline block copolymers in which the hard segment was composed of the additional long rigid-rod OPA blocks as a molecular fibre. Thus, the 
hard-segment acted as not only the physical crosslinks but also as a reinforced rigid-rod fibre in the soft-segment matrix to strongly enhance the tensile strength of the block copolymer as a molecular composite. The higher the long stiff aniline-oligomer blocks present in the block copolymer, the higher the tensile strength was observed. Earlier work [40] reported an initial increase in tensile strength resulting from the interpenetrating effect followed by a decrease due to the rigidity and brittleness of the PANI.

Stress- at-break and strain-at-break of electrically anisotropic PU-PANI samples were measured at constant rate of strain at room temperature by Špírková et al. [39] The temperature dependence of storage shear modulus was analysed at frequency of $1 \mathrm{~Hz}$. The ultimate mechanical properties, (stress- and strain-at-break, energy-to-break) were found to be considerably reduced in comparison to PU network prepared from triol, diisocyanate and catalyst. However, the storage modulus was found to be $2-5$ times higher as compared to the PU network at temperature range of $25-240^{0} \mathrm{C}$. A different work presented by Diaconu et al. [80] noted that tensile strength characteristics such as tensile stress and elongation at break decrease by incorporation of the polypyrrole who related the effects to enhanced phase mixing by electrostatic interactions of the polyurethane cationomer with oxidizing agents and polypyrrole.

\subsection{UV-Vis spectroscopy}

The UV-Vis absorption spectrum of diluted colloids of polythiophene containing urethane side chain measured by UV-Vis spectrophotometer using one-centimetre quartz cells at $25^{\circ} \mathrm{C}$ demonstrated solvatochromic behaviour [66]. A different study has been reported by Yang and Lee [37] who showed the absorption peaks of UV-Vis spectra of the films evacuated at $50^{\circ} \mathrm{C}$ for $24 \mathrm{~h}$ rising to 325-800 nm, which indicated that PANI was in the emeraldine salt form. In a recent work, Reece et al. [53] measured the UV-Vis spectra of diluted colloids using a UV-visible spectrophotometer and one centimetre path length cell. The UV-vis spectra obtained at different times during the growth of PPy/DBDA/PU colloidal dispersions contained a distinct peak centred at wavelengths above $800 \mathrm{~nm}$, signifying the presence of bipolaron charge carriers. Over the course of the reaction the peak above $800 \mathrm{~nm}$ shifted to lower wavelengths, indicating a decrease in the conjugation length and possibly some over-oxidation.

Malmonge et al. [38] reported UV-Vis-NIR spectroscopy analysis of the PU/PANI (99.8/0.2) blend doped in $1 \mathrm{M} \mathrm{HCl} / \mathrm{DMF}$ solutions within various times. The undoped blend showed a peak at 625 $\mathrm{nm}$ assigned to an excitation transition. A new absorption was noted at 410-450 nm after a much shorter time than in the case of doping in aqueous media though no further changes were observed 
at longer doping times in the electronic transitions. Complete doping was completely achieved only after about 320 hours.

\subsection{FT-IR spectroscopy}

Carone and et al. [77] covalently linked, by an amidation reaction, the terminal $\mathrm{NH}_{2}$ of emeraldine (EB) and sulfonated emeraldine (SPANI) to a free carboxylic group belonging to the repetitive unit of functionalized segmented polyurethane. The reaction was carried out by activating such a carboxylic group with $\mathrm{N}, \mathrm{N}^{\prime}$-dicyclohexylcarbodiimide and $\mathrm{N}$-hydroxysuccinimide. ${ }^{1} \mathrm{H}$ and ${ }^{13} \mathrm{C}$ NMR, UV, and FTIR studied the reaction course and the chemical properties of the polymers. The average numbers of EB or SPANI aromatic rings per polyether urethane acid repetitive unit, which cannot be assumed to be amidation degree because at this moment the molecular weights of the inserted EB and SPANI chains were unavailable, were six in case of the polymer obtained from the pristine emeraldine and one for that obtained from the sulfonated emeraldine. This result could be because SPANI was used in the acidic form, which depresses the nucleophilicity of the $\mathrm{NH}_{2}$ group because of the presence of the sulfonic protons. The p-conjugated ring orientation has been studied and compared for PANI against PPy by Choi et al. [84].

Different work [66] determined the IR spectra of the PU-P3UT at ambient temperature; samples were prepared by mixing urethane-substituted polythiophene powders with $\mathrm{KBr}$ to make pressed $\mathrm{KBr}$ pellets while in a previous work [65] the researchers casted thin films of the polymer and blends directly on a $\mathrm{KCl}$ plate from chloroform. Elsewhere, FT-IR characterisation of OPA and urethane-aniline block copolymers has been reported $[6,84,85]$.

\subsection{Morphology}

Morphological studies by Liao et al. [40] were performed using scanning electron microscopy (SEM) whereby the images were taken on the surface made by fracturing the specimen in liquid nitrogen and then casting it with Au powder. Scanning tunnelling microscopy (STM) images were obtained in air using a PtIr tip. The authors used conductive silver paint to make electric contacts with the samples. Scan speeds were kept at $\sim 3.8 \mathrm{~Hz}$ and the set point current data type was shown as computer generated topographical rendering. In the meantime, Wang et al. [36] used SEM to observe a thin bright layer of PANI-PU IPN in a relatively even thickness on the top of the bulk PU matrix. The layer was measured to be approximately $20 \mu \mathrm{m}$ in thickness and extended from the surface boundary of elastomer. 
Wen et al. [57], SEM took micrographs of TPU-PPy composites and pure TPU films by mounting polymer films on a metal substrate, surface was coated with carbon and then scanned in the microscope. Another SEM work presented scanning electron micrographs of unfilled and filled IPNs [15]. SEM micrograms of filled PU/PMMA IPN system showed spherulitic structure at higher concentration of polyaniline doped with camphor sulphonic acid (PANI-CSA).

Transmission electron microscopy (TEM) photographs of the PU-PANI matrix (5 wt.\% PANIPSSA) dried on a copper grid and stained with $2 \%$ phosphotungstic acid showed distinct globular structure with some interconnection among polyaniline particles [37]. However, the same team reported the conductivity of PU consisting of some aromatic groups but with the same PANI-PSSA concentrations showed interconnecting network structure, while those blends with 3 wt.\% PANIPSSA showed some isolated clusters, thus low connectivity between the clusters. The authors related this morphology to the conductivity in the interconnected network structure.

And lastly, Ruckenstein and Sun. [67] took the measurement of the PU films containing 3methylthiophene by first cutting the coated film and then magnifying with a microscope. The combined studies found that with increase in reaction time, the electrical resistance of the film decreased while the thickness of the coating layer increases.

\subsection{Thermal stability}

Differential scanning calorimetry (DSC) analysis was performed at a heating rate of $20^{\circ} \mathrm{C} / \mathrm{min}$ for the IPNs over a temperature range of -120 to $300^{\circ} \mathrm{C}$ by Liao et al. [40] and by Liu and Gregory $[65,66]$ on investigation of thermal stability of blends of PU and urethane substituted polythiophene in the $\beta$-position. The earlier found an increase of $T_{g}$ values with an increase of PANI content indicating the probability of permanent entanglement and interlocking significantly enhanced due to high compatibility when polyether-type UBMI are employed in the IPNs. The latter found the conducting composites to be of good thermal stability with $\mathrm{T}_{\mathrm{g}}$ and $\mathrm{T}_{\mathrm{m}}$ values of 62 and $265^{\circ} \mathrm{C}$, respectively. The thermal stabilities of PANI, PU/PMMA and PANI filled IPN systems were evaluated by thermogravimetry (TGA) too [42]. PU/PMMA IPNs were found stable up to $200^{\circ} \mathrm{C}$; further degradation up to completion at ca. $500^{\circ} \mathrm{C}$ undergoes in three-step process. In case of PANI filled IPN system, a two-step weight loss was observed and as well the pristine PANI could not degrade completely at this temperature.

The relative thermal stability of the PANI filled PU/PMMA IPNs were evaluated by comparing the decomposition temperatures at various percent weight losses [81] and integral procedural decomposition temperature (IPDT) which is an index of thermal stability of the system that was 
determined from the thermogram area using the method previously reported by Doyle [82]. It was observed that the initial thermal stability of PANI is enhanced after incorporating into PU/PMMA matrix. This is due to PANI entrenchment inside the PU/PMMA matrix, which drastically reduces moisture absorption behaviour of PANI. Other TGA work was of PPy/TPU composites [57] whereby TPU films and chemically prepared PPy were analysed over a temperature range of 100 $600^{\circ} \mathrm{C}$ in an inert atmosphere, at a heating rate of $20^{\circ} \mathrm{C} / \mathrm{min}$. The thermal stability of the composite was found to be comparable to pure TPU, though a decrease in $\mathrm{T}_{\mathrm{g}}$ of the soft segment was observed in the composite from DSC experiments. This may be due to interaction of PPy-NH groups with either carbonyl or ether oxygens of TPU, leading to phase separation of the hard and soft domains.

\section{Conclusions}

Polyurethane thermoplastic elastomers are extremely chemical resistant for use in the harshest environments whereby specially formulated PU meet unique requirements that are necessary for electronic and other material engineering applications. Conducting polymers such as polyaniline, polythiophene and polypyrrole exhibit excellent electrical conductivities and outstanding thermal stability, while their chemical solubility and mechanical properties are poor leading to a reduced processability. To overcome these difficulties most research efforts found in the literature were focused on the chemical functionalization of such conducting polymers and on composites of conducting polymers with elastomers, with thermoplastic polyurethanes being one of the most important group of materials.

It can be demonstrated that these polymers could be highly promising for many technological uses because of their chemical versatility, stability, processability and low cost. PU conducting composite/blends can be prepared in either chemical or electrochemical techniques with the aim of preparation of a new material combining synergistically the properties of the blend components. In the case of conducting polymers, the objective is the preparation of polymeric materials with good mechanical properties and processability associated with high conductivity or electrochromism. Both chemical and electrochemical techniques for preparation of polyurethane thermoplastic composites/blends have been briefly discussed. As well, various characterisation techniques utilised has been covered too. Improving the synthesis, characterisation techniques, conductivities and optimising the catalysts paves the way for the production of highly conductive materials with reproducible quality. 


\section{Acknowledgement}

This research has been supported by a Marie Curie Fellowship of the European Community programme "Improving the Human Research Potential and the Socio-Economic Knowledge Base" under Contract No. HPMT-CT-2001-00379. 


\section{References}

1. H. ISOTALO, M. AHLSKOG and H. STUBB, Synth. Met. 55-57 (1993) 3581.

2. Z. WIRPSZA, in "Poliuretany Chemia Technologia Zastosowanie" (Wydawnictwo Naukowo-Techniczne, Warsaw, 1991) p. 101.

3. R. PELRINE. R. KORNBLUH, J. JOSEPH, R. HEYDT, Q. PEI and S. CHIBA, Mater. Sci. Eng. C. 11 (2000) 89.

4. S. KOUL, R. CHANDRA and S. K. DHAWAN, Sensor. Actuat. B-Chem. 75 (2001) 151.

5. M. GERARD, A. CHAUBEY and B. D. MALHOTRA, Biosens. Bioelectron. 17 (2002) 345.

6. Y. Z. WANG, Y. C. HSU, R. R. WU and H. M. KAO, Synth. Met. 132 (2003) 151.

7. K. PIELICHOWSKI, Solid State Ionics. 104 (1997) 123.

8. P. PRON and P. RANNOU, Prog. Poly. Sci. 27 (2002) 135.

9. K. PIELICHOWSKI, J. PIELICHOWSKI. J. Therm. Analy. 53 (1998) 633.

10. K. PIELICHOWSKI and M. HASIK, Synth. Met. 89 (1997) 199.

11. D. KUMAR and R. C. SHARMA, Eur. Polym. J. 34 (1998) 1053.

12. S. S. RAY and M. OKOMOTO, Prog. Polym. Sci. 28 (2003) 1539.

13. N. GOSPODINOVA and L. TERLEMEZYAN, Prog. Polym. Sci. 23 (1998) 1443.

14. T. KANG, K. G. NEOH and K. L. TAN, Prog. Polym. Sci. 73 (1998) 277.

15. X. G. LI, M. R. HUANG, W. DUAN and Y. L.YANG, Chem. Rev. 102 (2002) 2925.

16. A. F. DIAZ, in "Organic Electrochemistry, An Introduction and Guide" (Marcel Dekker, New York, 1991) p.1363.

17. G. P. EVANS, in "Advances in Electrochemical Science and Engineering” (Wiley-VCH, Weinheim, 1990) p. 1.

18. J. M. MARGOLIS, in "Conductive Polymers and Plastics" (Chapman and Hall, New York, 1989) p. 1.

19. A. G. MACDIARMID and M. MAXFIELD, in "Electrochemical Science and Technology of Polymers" (Elsevier, London, 1987) p. 67.

20. G. K. CHANDLER and D. PLETCHER, in "Electrochemistry Specialist Periodical Report, Vol. 10" (Royal Society of Chemistry, London, 1986) p. 117.

21. L. DAI and A.W. H. MAU, J. Phys. Chem. B, 104 (2000) 1891.

22. A. DERONZIER and J. C. MOUTET, Acc. Chem. Res., 22 (1989) 249.

23. J. HEINZE, Top. Curr. Chem. 152 (1990) 1. 
24. M. D. IMISIDES, R. JOHN, P. J RILEY and G. G. WALLACE, Electroanalysis, 3 (1991) 879.

25. G. INZELT, M. PINERI, J. W. SCHULTZE and M. A. VOROTYNTSEV, Electrochimica Acta 45 (2000) 2403.

26. M. C. DE-JESUS, Y. FU and R. A. WEISS, Polym. Eng. Sci. 37 (1997) 1936.

27. J. E. OSTERHOLM, J. LAAKSO and P. NYHOLM, Synth. Met. 28 (1989) 435.

28. S. L. SHENOY, D. COHEN, C. ERKEY and R. A. WEISS, Ind. Eng. Chem. Res. 41 (2002) 1484.

29. P. BANERJEE, Eur. Polym. J. 34 (1998) 1557.

30. E. CSAHO'K, E. VIEIL and G. INZELT, Electroanal. Chem. 482 (2000) 168.

31. K. PIELICHOWSKI, J. PIELICHOWSKI, J. IQBAL and P.GURTAT, Appl. Catal. A: Gen. 161 (1997) L25.

32. K. S. HO, K. H. HSIEH, S. K. HUANG and T. H. HSIEH, Synth. Met.107 (1999) 65.

33. Y. CAO, P. SMITH, and A. HEEGER, Synth. Met. 48 (1992) 91.

34. Q. PEI and X. BI, J. Appl. Polym. Sci., 38 (1989) 1819.

35. T. SAWAI, H. SHINOHARA, Y. IKARIYAMA and M. J. AIZAWA, Electroanal. Chem. 283 (1990) 221.

36. L. Y. WANG, S. C. KUO and L. Y. CHIANG, Synth. Met. 84 (1997) 587.

37. S. M. YANG and H. L. LEE, Synth. Met. 102 (1999) 1226.

38. J. A. MALmONGE, C. S. CAMPOLI, L. F. MAlmONGE, D. H. F. KANDA, L. H. C. MATTOSO and G. O. CHIERICE, Synth. Met. 119 (2001) 87.

39. M. ŠPÍRKOVÁ, J. STEJSKAL and O. QUADRAT, Synth. Met., 102 (1999) 1264.

40. D. C. LIAO, K. H. HSIEH, Y. C. CHERN and K. S. HO, Synth. Met. 87 (1997) 61.

41. D. C LIAO and K. H. HSIEH, J. Polym Sci. Polym Chem. 32 (1994) 1665.

42. J. T. SIDDARAMAIAH, Eur. Polym. J. 39 (2003) 569.

43. Q. PEI and X. BI Synth. Met. 30 (1989) 351.

44. G. INZELT, “Electroanalytical Chemistry” (Marcel Dekker, New York, 1994) p. 89.

45. H. K. YOUSSOUFI, F. GARNIER, P. SRIVASTAVA, P. GODILLOT and A. YASSAR, J. Am. Chem. Soc. 119 (1997) 7388.

46. M. MORETA, I. HASHIDA and M. MISHIMURA, J. Appl. Polym. Sci. 36 (1988) 1639.

47. H.S.O. CHAN, T.S.A. HOR, P.K.H. HO, K.L. TAN and B. T. G. TAN, J. Macromol. Sci. Chem. 27 (1990) 1081.

48. S. E. LINDSEY and G. B. STREET, Synth. Met. 10 (1984) 67.

49. O. NIWA, M.KAKUCHI and T. TAMAMURA, Synth. Met. 18 (1987) 677.

50. C. LI and Z. SONG, Synth. Met. 40 (1991) 23. 
51. R. M. PENNER and C. R. MARTIN, J. Electrochem. Soc. 33 (1986) 310.

52. M. WATANABE, H. SHIRAI and T. HIRAI, J. Appl. Phys. 92 (2002) 4631.

53. D. A. REECE, P. C. INNIS, S. F. RALPH and G. G. WALLACE, Colloid. Surface. A. 207 (2002) 1 .

54. A. E. WIERSMA, L. M. A. VAN STEEG and T. J. M. JONGELING, Synth. Met. 71 (1995) 2269.

55. B. ZINGER, Synth. Met. 28 (1989) 37.

56. J. ZINGER, Electroanal. Chem. 244 (1988) 115.

57. T. C. WEN, S. L. HUNG and M. DIGAR, Synth. Met.118 (2001) 11.

58. X. BI and Q. PEI, Synth. Met. 22 (1987) 145.

59. S. L. SHENOY, I. KAYA, C. ERKEY and R. A. WEISS, Synth. Met. 123 (2001) 509.

60. Y. FU, R. A. WEISS, P. P. GAN, M. D. BESSETTE, J. Polym. Eng. Sci. 38 (1998) 857.

61. F. HE, M. OMOTO, T. YAMAMOTO and H. KISE, Kobunshi Ronbunshu 50 (1993) 653.

62. F. HE, M. OMOTO, T. YAMAMOTO and H. KISE, Nippon Kaguka Kaishi, 3 (1993) 296.

63. A. E. WIERSMA and L. M. A. VAN STEEG, European Pat. No. 589529 (1994).

64. B. SARI, M. TALU, F. YILDIRIM and E. K BALCI, J. Appl. Surf. Sci. 205 (2003) 27.

65. R. V. GREGORY and M. LIU, Synth. Met. 69 (1995) 349.

66. R. V. GREGORY and M. LIU, Synth. Met. 72 (1995) 45.

67. E. RUCKENSTEIN and Y. SUN, Synth. Met. 75 (1995) 79.

68. G. HEYWANG, F. JONAS, Adv. Mater. 4 (1992) 116.

69. Q. PEI, G. ZUCCARELLO, M. AHLSKOG and O. INGANAS, Polymer 35 (1994) 1347.

70. A. KUMAR and J. R. REYNOLDS, Macromolecules 29 (1996) 7629.

71. M. A. KHAN and S. P. ARMES, Langmuir 15 (1999) 3469.

72. A. R. SANJAI, T. S. NATARAJAN, G. RANGARAGAN, S. THOMAS, P. V. PRAVAKARAN and S. VENKATACHALAM, Phys. Rev. B 55 (1997) 10734.

73. M. CHIPARA, D. HUI, P. V. NOTINGHER, M. D. CHIPARA, K. T. LAUE, J. SANKARF and D. PANAITESCU, Composites: Part B 34 (2003) 637.

74. B. SIXOU, N. MERMILliOD, J. P. TRAVERS, Phys. Rev. B 53 (1996) 4509.

75. N. F. MOTT and E. A. DAVIES, "Electronic Processes in Non-Crystalline materials" (Oxford University Press, Oxford, 1979) p. 13.

76. P. DUTTA, S. BISWAS, M. GHOSH, S. K. DE and S. CHATTERJEE, Synth. Met. 122 (2001) 455.

77. E. CARONE, L. D'IlARiO and A. MARTinelli, J. Appl. Polym. Sci. 83 (2002) 857.

78. E. CARONE, L. D'ILARIO and A. MARTINELLI, J. Appl. Polym. Sci. 86 (2002) 1259. 
79. L. I. SOLIMAN, W. M. SAYED, T. A. HENDIA and E. A. ISMAIL, Phys. Low Dimens. Struct. 11-12 (2002) 119.

80. I. DIACONU, P. COMAN, T. BURUIANA and E. BURUIANA, in Proceedings of the $10^{\text {th }}$ International Symposium on Electrets (ISE 10) Delphi, Greece, September 1999, edited by A. A. Konsta, A. Vassilikou-Dova, K. Vartzeli-Nikaki (IEEE, Piscataway, NJ, USA, 1999) p. 493.

81. R. K. JAIN, L. KRISHNAN and H. L. BHATNAGAR, J Polym Mater. 6 (1989) 287.

82. C. D. DOYLE, Anal. Chem. 33 (1961) 77.

83. T.J. SKOTHEIM, "Handbook of Conducting Polymers" (Marcel Dekker, New York, 1986) p.1.

84. J. CHOI, M. CHIPARA, B. XU, C. S. YANG, B. DOUDIN and P. A. DOWBEN, Phys. Chem. Lett. 343 (2001) 193.

85. D. S. PARK and Y. SHIM, Electrochem. Soc. 140 (1993) 609.

86. S. MU and J. KAN, Synth. Met. 98 (1998) 51. 


\section{Caption for Tables and Figures}

\section{Tables}

Tab. I. Structure and molecular masses of selected diisocyanates.

Tab. II. Comparison in conductivity of some conjugated polymers

Tab. III. Synthesis and characterisation of PU composites/blends

Tab. IV. Conducting properties of PU composites/blends

\section{Schemes}

Sch. 1. Schematic structure of segmented polyurethane. Adopted from Wirpsza [2].

Sch. 2. Chosen reaction path for preparation of a PU prepolymer. Adopted from Wang et al. [6].

Sch. 3. Representation of hydrogen bond formation between -NH of PANI and -NHCO group of PU. Adopted from Siddaramaiah [42]

\section{Figures}

Fig. 1. Technological applications of conductive polymers. Adopted from Kumar and Sharma [11]. 
Table I

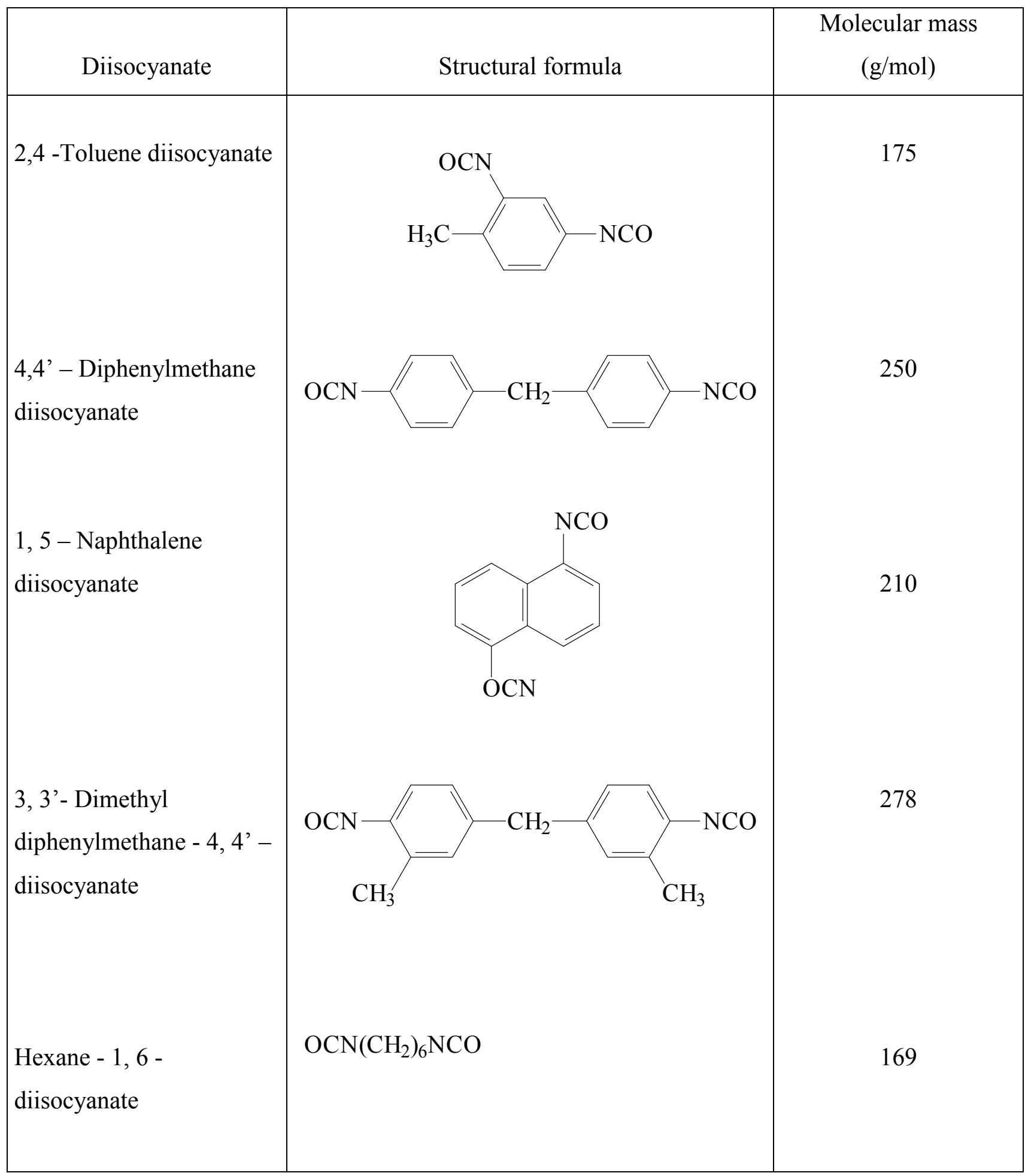


Table II

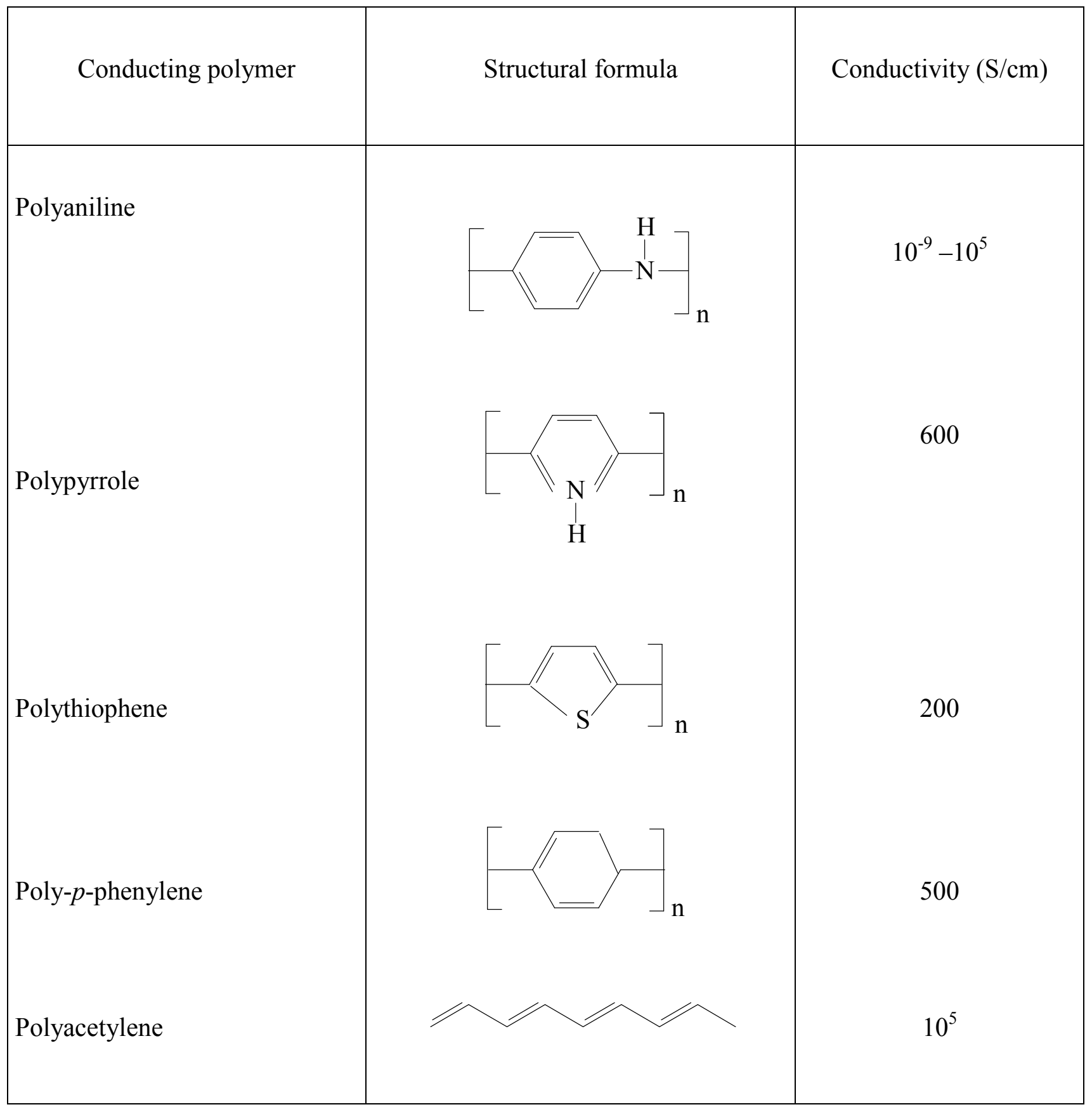


Table III

\begin{tabular}{|c|c|c|c|}
\hline $\begin{array}{l}\text { Conducting } \\
\text { polymer }\end{array}$ & Synthetic Process & Characterisation technique & Ref. \\
\hline PANI & Chemical oxidation & SEM, X-ray scattering & 36 \\
\hline PANI & Chemical oxidation & SEM, TEM, UV-Vis & 37 \\
\hline PANI & Chemical oxidation & Conductivity, UV-Vis, NMR & 38 \\
\hline PANI & Electrochemical & Mechanical properties, conductivity & 39 \\
\hline PPY & Chemical oxidation & Conductivity & 54 \\
\hline PANI & Chemical oxidation & $\begin{array}{l}\text { FT-IR, conductivity, }{ }^{13} \mathrm{C} \quad \mathrm{CP} / \mathrm{MAS} \quad \mathrm{NMR}, \text { mechanical } \\
\text { properties }\end{array}$ & $2,40,41$ \\
\hline PANI & Chemical oxidation & DSC, SEM, STM, conductivity, mechanical properties & 40 \\
\hline PMT & Chemical oxidation & Morphology, conductivity, mechanical properties & 67 \\
\hline PANI & Chemical oxidation & $\begin{array}{l}\text { TGA, SEM, chemical resistivity, electrical-, physical- and } \\
\text { mechanical - properties }\end{array}$ & 42 \\
\hline PPy & Electrohydrodynamic & UV-Vis, photon correlation spectroscopy (PCS) & 53 \\
\hline PPy & Chemical oxidation & SEM, TGA, swelling studies, conductivity & 57 \\
\hline PPy & Electrochemical & $\begin{array}{l}\text { Cyclic voltammograms, FT-IR, SEM, mechanical and } \\
\text { electrical properties }\end{array}$ & 58 \\
\hline PPy & Chemical oxidation & Conductivity & 60 \\
\hline PPy & Chemical oxidation & Conductivity & 59 \\
\hline PPy & Chemical oxidation & SEM, conductivity & 28 \\
\hline Polythiophene & Chemical oxidation & $\begin{array}{l}\text { DSC, DMTA, UV-Vis, }{ }^{13} \mathrm{C} \text { NMR, FT-IR, SEM, morphology, } \\
\text { conductivity }\end{array}$ & 66 \\
\hline Polythiophene & Chemical oxidation & ${ }^{13} \mathrm{C} /{ }^{1} \mathrm{H}$ NMR, FT-IR, elemental analysis, MS & 65 \\
\hline PANI & Chemical oxidation & Conductivity, magnetism, thermal stability, transport properties & 72 \\
\hline PANI & Electrochemical & FT-IR, optical properties & 43 \\
\hline
\end{tabular}


Table IV

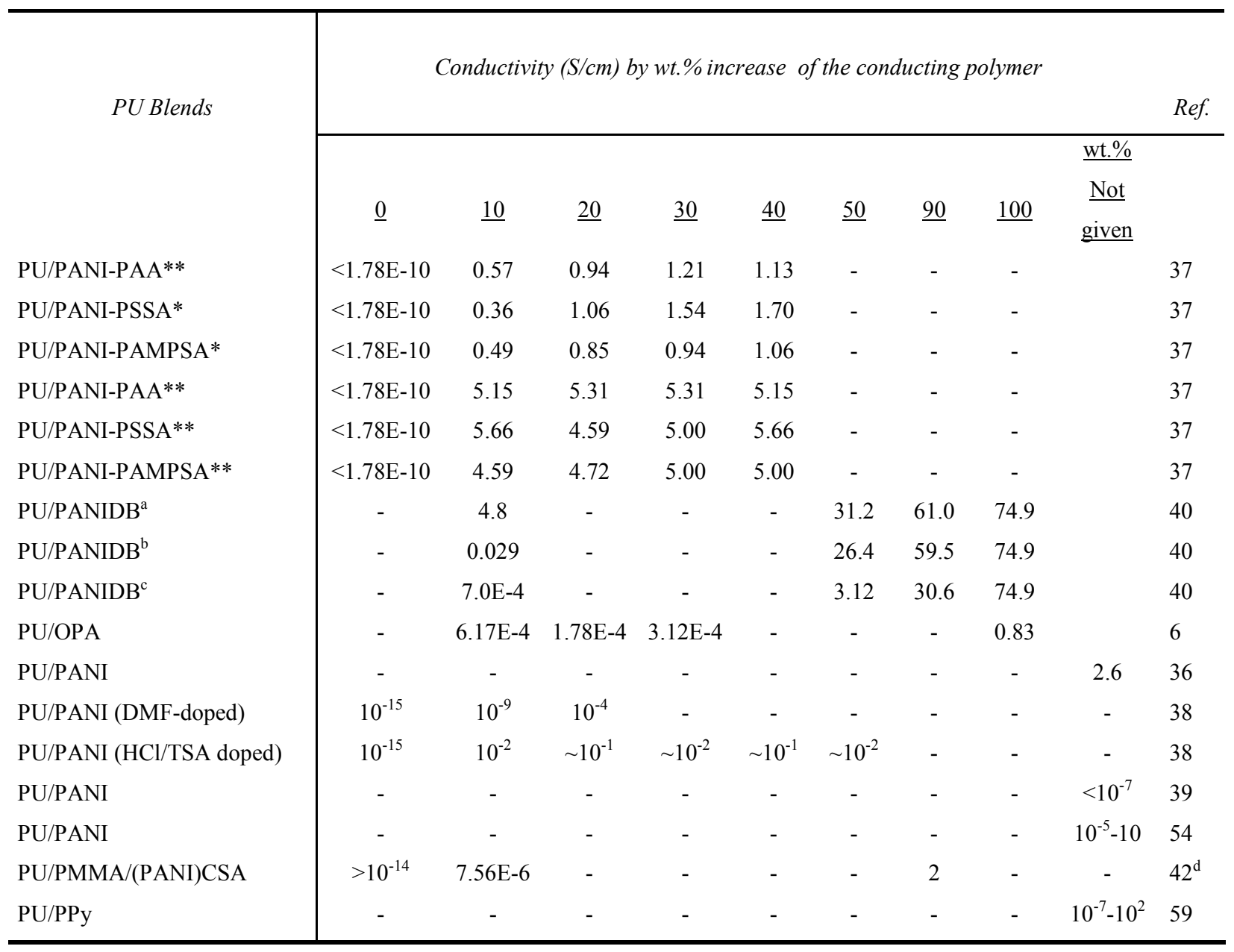

Notes: *aliphatic PU, ** aromatic PU. Poly(tetramethylene oxide) with molecular weight 650 (PMTO 650) (), 1000 (PMTO 1000) $\left({ }^{b}\right)$ and poly(butylene adipate) (PBA) with molecuar weight 1000 (PBA 1000) $\left({ }^{c}\right)$ were used as polyols. $\left({ }^{\mathrm{d}}\right)$ electrical properties of (PANI)CSA filled PU/PMMA (50/50) IPN. 
Scheme 1.

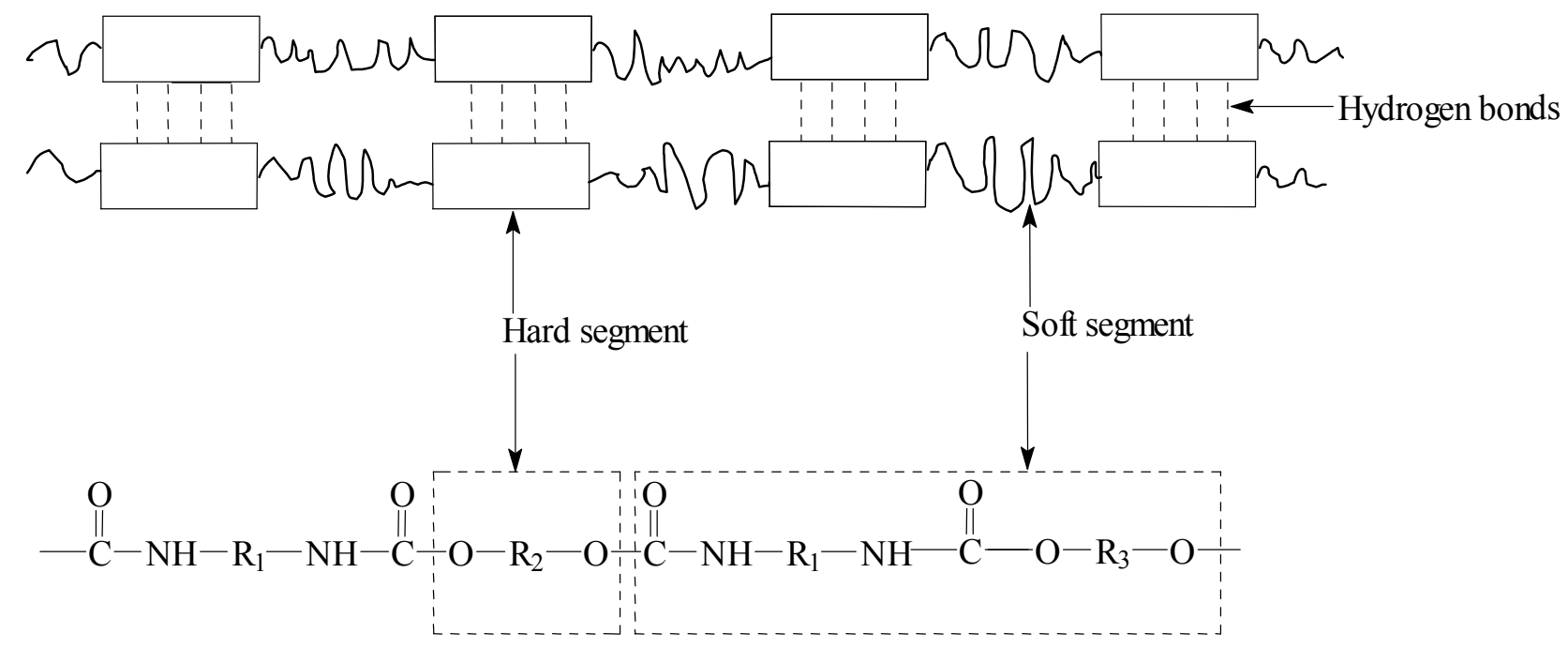




\section{Scheme 2}

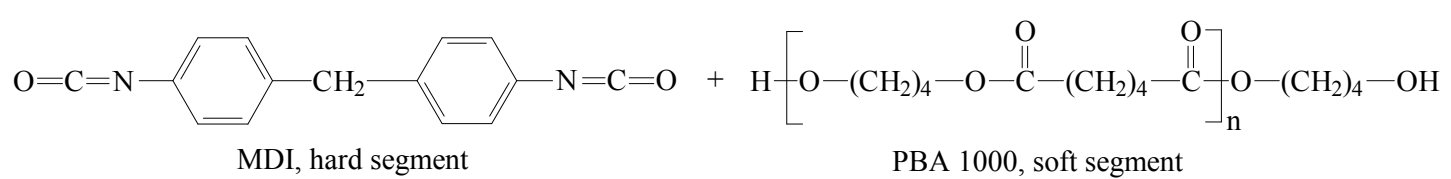

PBA 1000, soft segment

Under dried $\mathrm{N}_{2}$

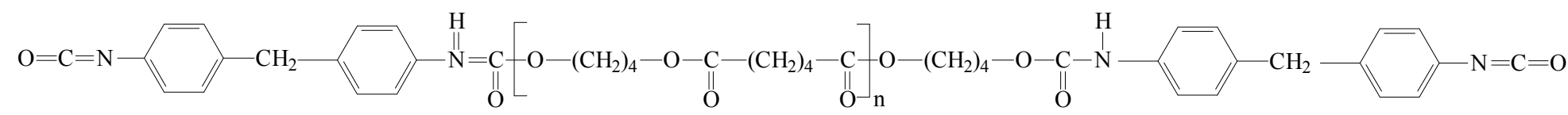

Chain extender, under dried $\mathrm{N}_{2}$

OPA/1,4-BD in NMP

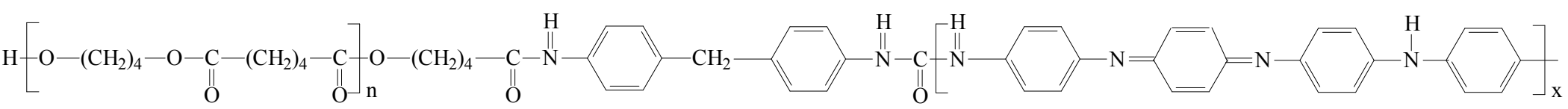


Scheme 3.

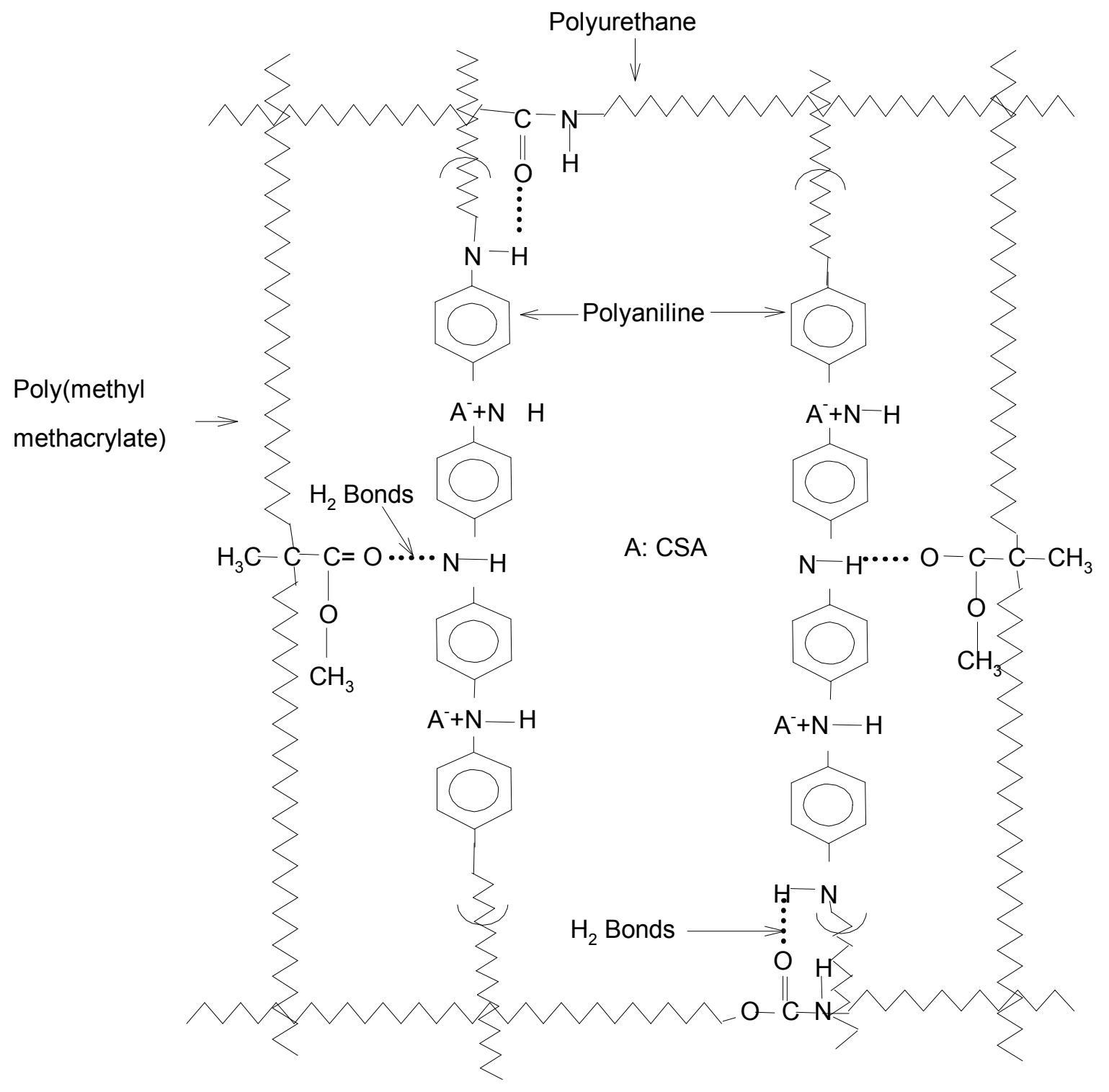


Fig.1

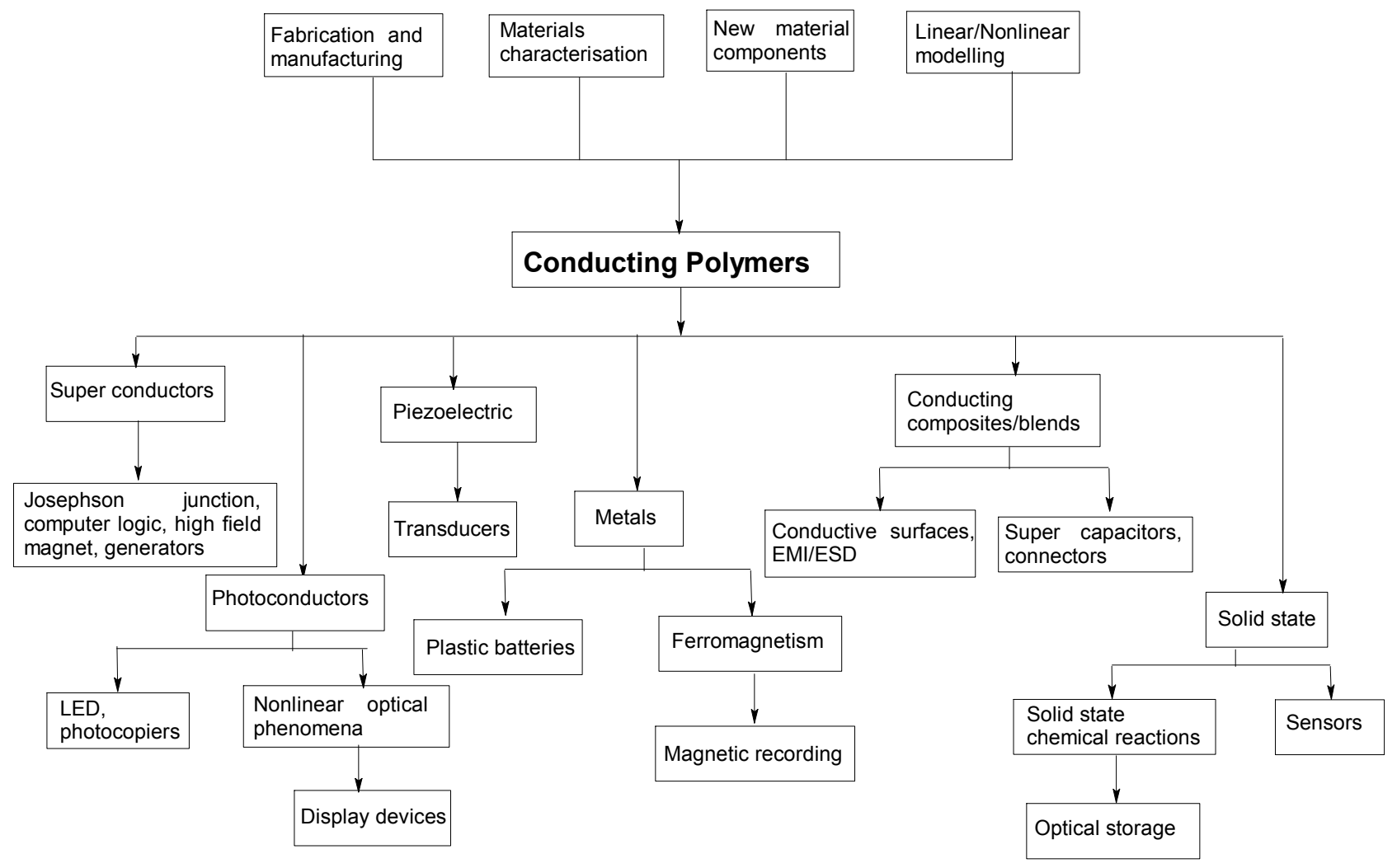

